Exp. Anim. 55(4), 399-404, 2006

-Note-

\title{
Morphometric Study of Gender Differences with Regard to Age-Related Changes in the C57BL/6 Mouse Kidney
}

\author{
Akira YABUKI'), Shin TANAKA ${ }^{2)}$, Mitsuharu MATSUMOTO1), \\ and Shusaku SUZUKI ${ }^{1)}$
}

\begin{abstract}
1) Laboratory of Veterinary Anatomy, Department of Veterinary Medicine, Faculty of Agriculture, Kagoshima University, 1-21-24 Korimoto, Kagoshima 890-0065, and 2) Animal Facilities for Aging Research, National Institute for Longevity Sciences (NILS), National Center for Geriatrics and Gerontology, 36-3 Gengo Morioka-cho, Obu 474-8522, Japan
\end{abstract}

\begin{abstract}
To investigate gender differences with regard to age-related changes in the mouse kidney, kidneys from male and female C57BL/6Cr mice were morphometrically examined at 2, 4, 12, 27 and 33 months of age. Gender differences were clearly observed in age-related changes in renal corpuscle diameter, in addition to semiquantitative scores of glomerular damage and interstitial fibrosis. A greater relative increase in renal corpuscle diameter was observed in females compared to males, and scores indicating glomerular damage and interstitial fibrosis were more severe in males than in females.
\end{abstract}

Key words: aging, gender differences, mouse kidney

Several types of nephropathy progress more rapidly in men than in women $[9,10,15]$. The mouse is the most commonly used rodent for laboratory experiments, and investigations as to whether the course of nephropathy differs between male and female mice would represent a large contribution toward clarifying the pathophysiological mechanisms of gender differences in the progression of nephropathy. However, gender differences with regard to age-related renal changes have not been investigated in mice. In particular, morphometric data is lacking. The present study was thus designed to investigate the kidneys of $\mathrm{C} 57 \mathrm{BL} / 6 \mathrm{Cr}$ mice, a standard background strain for the production of transgenic mice, from 2 to 33 months of age using a morphometric approach.

Male and female C57BL/6CrSlc mice were used in the present study at 2, 4, 12, 24 and 33 months of age $(\mathrm{n}=3$ per age group). All animal experiments were approved by the Committee for Animal Ethics of the National Institute for Longevity Science (NILS), based on NILS guidelines for animal experimentation in consideration of animal rights and welfare. Mice were housed in an animal room (temperature $22-23^{\circ} \mathrm{C}$; light exposure, 08:00-20:00) at the NILS Aging Farm, dur-

(Received 6 January 2006 / Accepted 27 February 2006)

Address corresponding: A. Yabuki, Laboratory of Veterinary Anatomy, Department of Veterinary Medicine, Faculty of Agriculture, Kagoshima University, 1-21-24 Korimoto, Kagoshima 890-0065, Japan 
ing which time Labo MR Stock (Nihon Nousan, Yokohama, Japan), a low-protein diet (18\% protein), was provided for long life, and water was supplied ad libitum [16]. All mice were sacrificed by administration of a lethal dose of 2, 2, 2-tribromoethanol (Avertin), after which the kidneys were quickly removed and weighed. Central slices from the kidneys, including the hilum, were cut perpendicular to the long axis and fixed in $10 \%$ neutral buffered formalin. After routine embedding in paraffin, $3 \mu \mathrm{m}$-thick sections were selected every $30 \mu \mathrm{m}$ and stained with hematoxylin-eosin, periodic acid Schiff (PAS) and Masson's trichrome (MT). Randomized morphometric analyses were conducted using 5 sections from each animal. The diameter of each renal corpuscle was then evaluated. A total of 5 cortical renal corpuscles with a vascular or urinary pole were selected from each PAS-stained section. A line from the vascular to the urinary pole was taken as the vertical axis, and the widest distance along this axis was taken as the renal corpuscle diameter. The extent of glomerular damage was then evaluated using a previously described semiquantitative scoring system [12]. A minimum of 250 glomeruli per animal were examined in MT-stained sections, and the severity of glomerular damage as indicated by endothelial or mesangial cell proliferation, or by expansion of the mesangial matrix, was graded from 0 to +4 according to the relative proportion of glomeruli involved: 0 , normal; $+1, \leq 25 \%$ damaged; $+2,26-50 \%$ damaged; +3 , $51-75 \%$ damaged; and $+4,76-100 \%$ damaged. The glomerular damage score was evaluated for each animal. If, for example, 10 of 100 glomeruli displayed +1 lesions, 20 of 100 demonstrated +2 lesions, 15 of 100 demonstrated +3 lesions, and five of 100 demonstrated +4 lesions, the final sclerosis score would be: $[(1 \times 10 /$ $100)+(2 \times 20 / 100)+(3 \times 15 / 100)+(4 \times 5 / 100)]$ $\times 100=115$. The extent of interstitial fibrosis was also evaluated using a similar semiquantitative scoring system. A minimum of 40 non-overlapping cortical fields (magnification $\times 200$ ) per animal were examined in MT-stained sections, and the severity of fibrosis was graded from 0 to +4 : 0 , normal; +1 , mild fibrosis; +2 , moderate fibrosis; +3 , severe fibrosis; and +4 , very severe fibrosis. Interstitial fibrosis score was evaluated for each animal. If, for example, five of 20 fields displayed +1 lesions, three of 20 demonstrated +2 lesions, two of 20 demonstrated +3 lesions, and one of
20 demonstrated +4 lesions, the final fibrosis score would be: $[(1 \times 5 / 20)+(2 \times 3 / 20)+(3 \times 2 / 20)+(4 \times$ $1 / 20)] \times 100=105$. The results were expressed as the mean \pm standard error of the mean and analyzed statistically using nonparametric methods $(P<0.05)$. The Mann-Whitney U-test was used to compare the results of males and females in each age group. The KruskalWallis test was used to evaluate age-dependent changes in each sex.

All quantitative data are shown in Fig. 1. Body weight (BW) increased up to 12 and 24 months, and decreased thereafter, in males and females, respectively (Fig. 1A). Age-dependent changes in BW were significant in both males and females. Gender differences were detected in each age group, apart from mice aged 24 months, with males exhibiting significantly greater BW than females. Total kidney weight (TKW) of males increased up to 24 months, after which a decline in TKW was observed (Fig. 2B). Females demonstrated similar changes. Age-dependent changes in TKW were significant in both males and females. Gender differences were detected in each age group, apart from mice at 33 months of age, with males exhibiting significantly greater TKW than females. Age-dependent changes in the ratio of TKW/BW were not significant in males or females (Fig. 1C). Renal corpuscle diameter increased in males up to 24 months, after which time a slight decrease in renal corpuscle diameter was observed (Fig. 1D). Females demonstrated similar changes in renal corpuscle diameter. Age-related changes in renal corpuscle diameter were significant in both males and females. Gender differences were detected at 2, 4 and 12 months of age, with males exhibiting significantly greater values than females. Figure 2 shows the results of light microscopic examination of kidneys from 33month-old males. Within the glomeruli, thickening of the glomerular basement membrane was observed, in addition to proliferation of endothelial and mesangial cells, and expansion of the mesangial matrix (Fig. 2). Within the tubulointerstitium, thickening of the tubular basement membrane, tubular atrophy, urinary casts, mononuclear cell infiltration and increased connective tissue were observed (Fig. 2). Similar histopathological observations were made in males and females, peaking in severity at 24 and 33 months of age. Glomerular damage scores increased at 12 months of age, and peaked at 24 and 33 months of age in males and 
females, respectively (Fig. 1E). Age-related changes in glomerular damage scores were significant in both males and females. Gender differences were detected at 4 , 12, 24 and 33 months of age, with males exhibiting significantly greater scores than females. Interstitial fibrosis scores increased at 12 months of age, and remained elevated up to 33 months of age, in both males and females (Fig. 1F). Age-related changes in interstitial fibrosis scores were significant in both males and females. Gender differences were detected at 24 and 33 months of age, with males exhibiting significantly greater scores than females.

The present study demonstrates gender differences in the progression of age-related renal changes in C57BL/6Cr mice. With regard to kidney size, although age-dependent increases in TKW were observed, significant changes in the ratio of TKW/BW were not observed in males or females. Renal hypertrophy thus does not represent a feature of age-related changes in C57BL/6Cr mice.

The increase in renal corpuscle diameter observed in this study suggests glomerular hypertrophy. Specifically, from 12 to 24 months of age, a markedly greater relative increase in renal corpuscle diameter was observed in females, compared to males, suggesting that glomerular hypertrophy is more severe in females than males during this period. Several studies have described close relationships between glomerular hypertrophy and glomerulopathy in aging mice and rats [4, 13, 19, 22], however, no direct relationship between the two events has been reported [2, 14, 17]. In the present study, similar age-related increases in both renal corpuscle diameter and glomerular damage scores were observed, particularly in males. Gender differences with regard to severity of age-related changes in these parameters were observed when comparing males and females. Briefly, a greater relative increase in renal corpuscle diameter was observed in females than males, while glomerular damage scores were greater in males than females. Moreover, changes in diameter of renal corpuscles may have been affected not only by changes in glomerular size, but also by changes in height of the parietal layer of glomerular capsules. Actually, the presence of a cuboidal parietal layer in the glomerular capsules represents a well-known morphological feature of the adult male mouse kidney, and the relationship between the diameter of renal corpuscles and the height of the parietal layer of glomerular capsules has already been suggested [18]. The present study failed to demonstrate any association between increased renal corpuscle diameter and glomerular damage in the course of age-dependent nephropathy. In addition, although reductions in nephron number are known to induce glomerular hypertrophy and damage [4], age-dependent reductions in nephron number could not be assessed in the present study.

The rate of deterioration in renal function is now widely accepted as strongly correlating with degree of tubulointerstitial nephritis $[1,5,21]$. Histopathological features of tubulointerstitial nephritis, including mononuclear cell infiltration, tubular atrophy and interstitial fibrosis, were clearly observed in aged mice in the present study. Interstitial fibrosis scores increased from 12 to 33 months of age without reaching a plateau in both males and females in the present study, and a greater relative increase in score was observed in males. Tubulointerstitial nephritis with interstitial fibrosis might thus correlate with age-related deterioration in renal function during the later stages of life in C57BL/ $6 \mathrm{Cr}$ mice, and age-related nephropathy may be more severe in males than in females.

In conclusion, the present study demonstrated gender differences in the progression of age-related nephropathy in $\mathrm{C} 57 \mathrm{BL} / 6 \mathrm{Cr}$ mice. Although a greater increase in renal corpuscle diameter was observed in females than in males, glomerular damage and interstitial fibrosis were more severe in males than in females. The protective effects of estrogen and the exacerbating effects of androgens, which affect the activities of mediators such as nitric oxide synthases, matrix metalloproteinases and transforming growth factor- $\beta 1$, on the course of nephropathy have already been demonstrated in several studies $[6-8,11,20]$. The gender differences demonstrated in glomerular damage and interstitial fibrosis in the present study might also be caused by the effects of sexual hormones. However, the greater increase of renal corpuscle diameter in females cannot be explained by the effects of sexual hormones, as described above. Further studies are thus needed to clarify the pathophysiological mechanisms underlying gender differences in the progression of age-dependent nephropathy. 

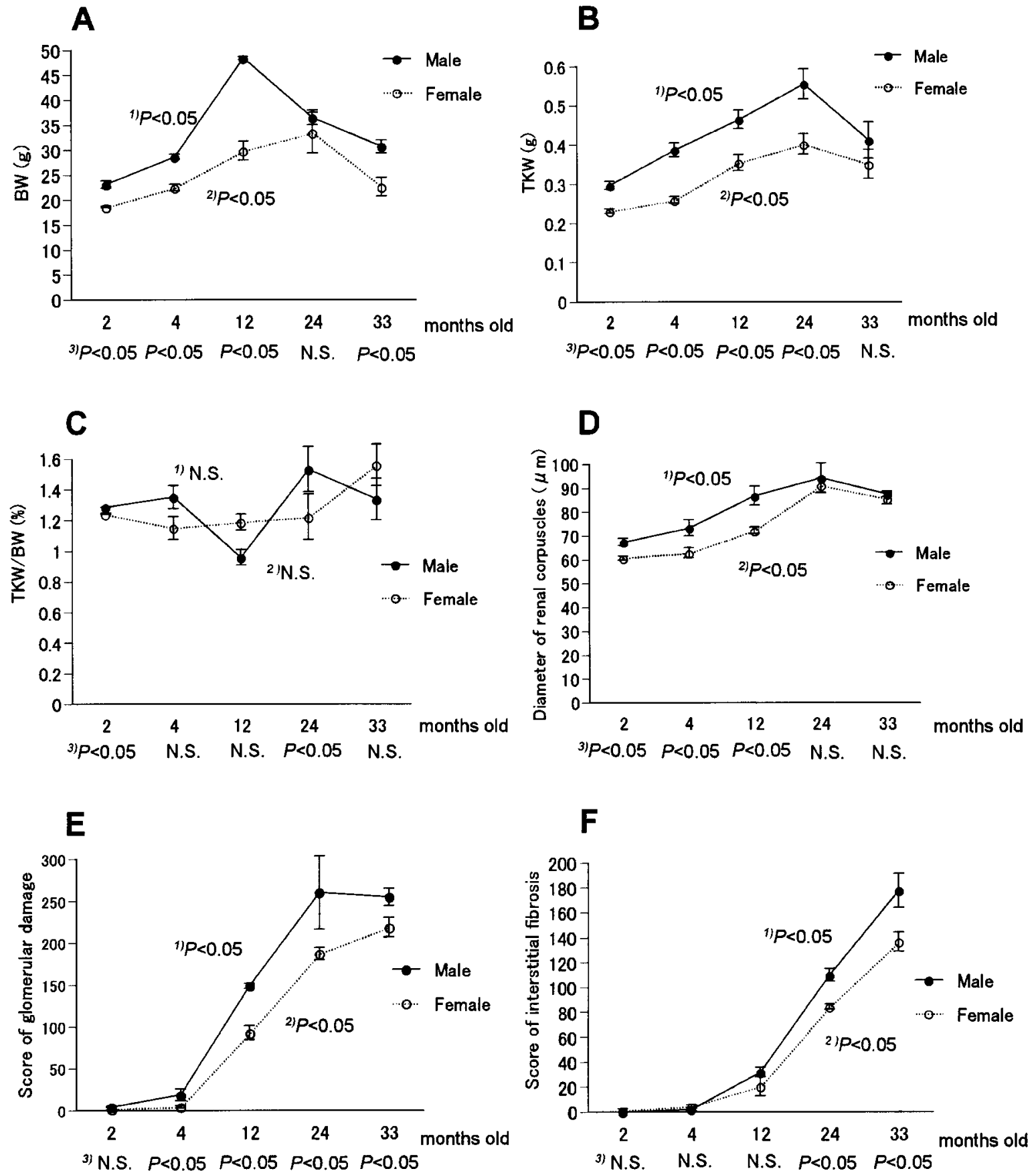

Fig. 1. Changes in body weight, kidney weight and morphometric parameters. (A) Body weight (BW). (B) Total kidney weight (TKW). (C) Ratio of TKW/BW. (D) Diameter of renal corpuscles. (E) Score of glomerular damage. (F) Score of interstitial fibrosis. Each column represents mean \pm SEM. ${ }^{1}$ Significant changes in male mice (Kruskal-Wallis test). ${ }^{2}$ Significant changes in female mice (Kruskal-Wallis test). ${ }^{3}$ Statistical differences between males and females in each age group (Mann-Whitney U test). N.S.: not significant. 

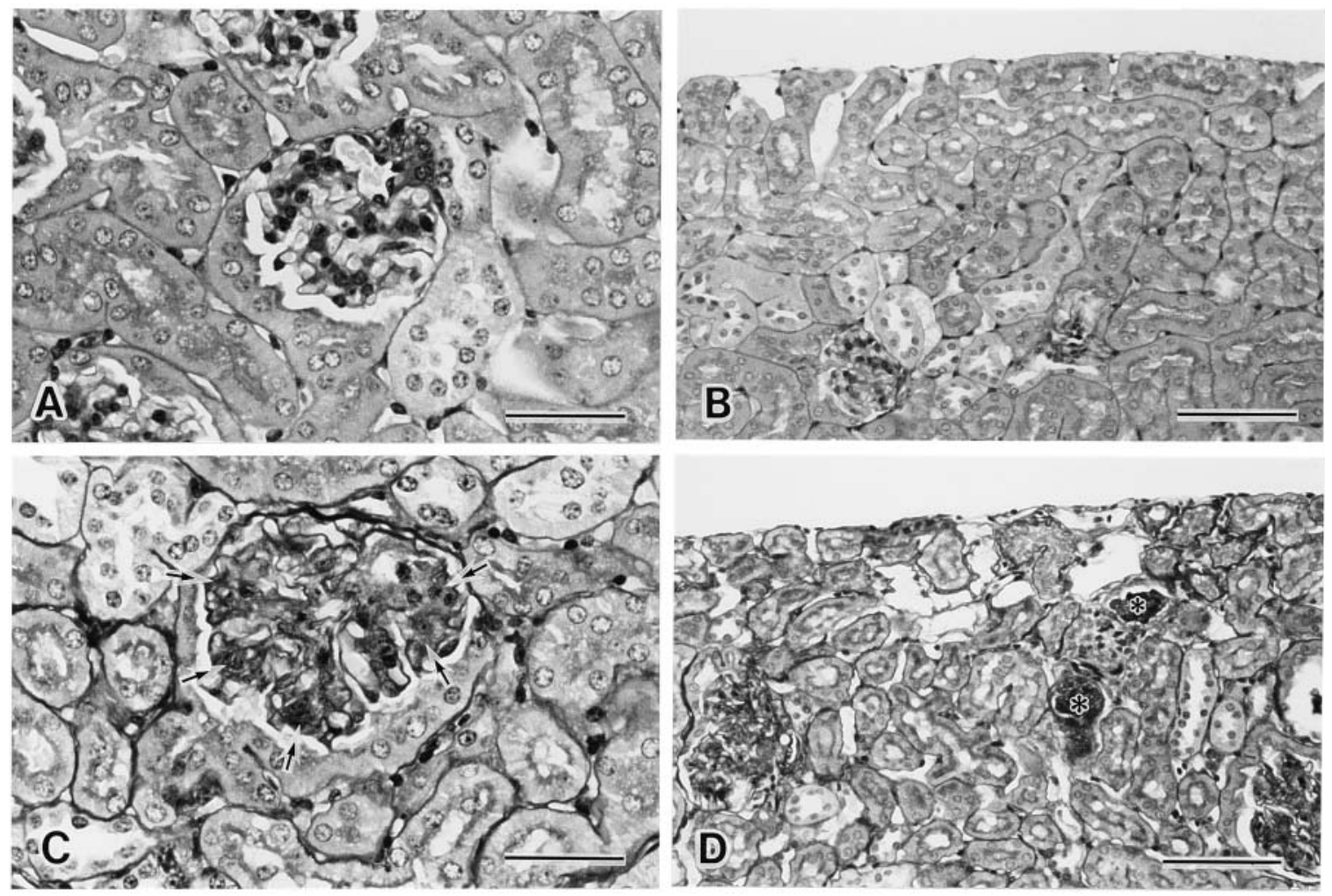

Fig. 2. Light micrographs of renal cortex from 4- and 33-month-old male mice. (A) Renal corpuscle at 4 months of age. (B) Tubulointerstitium at 4 months of age. (C) Renal corpuscle at 33 months of age. (D) Tubulointerstitium at 33 months of age. At 4 months of age, no lesions are observed within the renal corpuscles (panel A) and tubulointerstitium (panel B). At 33 months of age, glomerular damage with thickening of the glomerular basement membrane, proliferation of endothelial and mesangial cells and expansion of the mesangial matrix, are observed within the renal corpuscles (arrows, panel C), and thickening of the tubular basement membrane, tubular atrophy, urinary casts (asterisks), mononuclear cell infiltration, and increased connective tissue, are observed within the tubulointerstitium (panel D). PAS stain. Scale bars, $40(\mathrm{~A} \& \mathrm{C})$ and $80(\mathrm{~B} \& \mathrm{D}) \mu \mathrm{m}$.

\section{References}

1. Alexopoulos, E., Seron, D., Hartley, R.B., and Cameron, J.S. 1990. Kidney Int. 37: 100-109.

2. Baylis, C. 1994. J. Clin. Invest. 94: 1823-1829.

3. Erdely, A., Greenfeld, Z., Wagner, L., and Baylis, C. 2003. Kidney Int. 63: 1021-1026.

4. Fogo, A. and Ichikawa, I. 1991. Am. J. Kidney Dis. 17: 666-669.

5. Gilbert, R.E. and Cooper, M.E. 1999. Kidney Int. 56: 16271637.

6. Kwan, G., Neugarten, J., Sherman, M., Ding, Q., Fotadar, U., Lei, J., and Silbiger, S. 1996. Kidney Int. 50: 11731179.

7. Maric, C., Sandberg, K., and Hinojosa-Laborde, C. 2004. J. Am. Soc. Nephrol. 15: 1546-1556.

8. Negulescu, O., Bognar, I., Lei, J., Devarajan, P., Silbiger, S., and Neugarten, J. 2002. Kidney Int. 62: 1989-1998.

9. Neugarten, J. 2002. J. Am. Soc. Nephrol. 13: 2807-2809.
10. Neugarten, J., Acharya, A., and Silbiger, S.R. 2000. J. Am. Soc. Nephrol. 11: 319-329.

11. Park, K.M., Kim, J.I., Ahn, Y., Bonventre, A.J., and Bonventre, J.V. 2004. J. Biol. Chem. 279: 52282-52292.

12. Raij, L., Azar, S., and Keane, W. 1984. Kidney Int. 26: 137-143.

13. Sanden, S.K., Wiggins, J.E., Goyal, M., Riggs, L.K., and Wiggins, R.C. 2003. J. Am. Soc. Nephrol. 14: 2484-2493.

14. Sato, F., Tsuchihashi, S., and Kawashima, N. 1975. Exp. Gerontol. 10: 325-331.

15. Silbiger, S.R. and Neugarten, J. 1995. Am. J. Kidney Dis. 25: 515-533.

16. Tanaka, S., Segawa, T., Tamaya, N., and Ohno, T. 2000. Arch. Gerontol. Geriatr. 30: 215-223.

17. Yabuki, A., Matsumoto, M., Nishinakagawa, H., and Suzuki, S. 2003. J. Vet. Med. Sci. 65: 845-851.

18. Yabuki, A., Suzuki, S., Matsumoto, M., and Nishinakagawa, H. 1999. J. Vet. Med. Sci: 61: 891-896.

19. Yabuki, A., Tanaka, S., Matsumoto, M., Kamimura, R., 
and Suzuki, S. 2004. Kyushu J. Exp. Anim. 20: 9-14.

20. Verzola, D., Gandolfo, M.T., Salvatore, F., Villaggio, B., Gianiorio, F., Traverso, P., Deferrari, G., and Garibotto, G. 2004. Kidney Int. 65: 1252-1261.

21. Wehrmann, M., Bohle, A., Bogenschutz, O., Eissele, R.,
Freislederer, A., Ohlschlegel, C., Schumm, G., Batz, C., and Gartner, H.V. 1989. Clin. Nephrol. 31: 67-76.

22. Zheng, F., Plati, A.R., Potier, M., Schulman, Y., Berho, M., Banerjee, A., Leclercq, B., Zisman, A., Striker, L.J., and Striker, G.E. 2003. Am. J. Pathol. 162: 1339-1348. 